\title{
QBCO and NSQBCO Based Multi-User Single-Relay Selection Scheme in Cooperative Relay Networks
}

\author{
Jizheng $\mathrm{Li}^{1,2}$ and Ming Diao ${ }^{1}$ \\ ${ }^{1}$ School of Information and Communication Engineering, Harbin Engineering \\ University, Harbin, 150001, China \\ ${ }^{2}$ Heilongjiang Province Key Lab of Measurement-Control Tech and Instrument, \\ Harbin University of Science and Technology, Harbin, 150080, China \\ lijizheng@hrbust.edu.cn
}

\begin{abstract}
In cooperative relay networks with multiple users and multiple potential relays, which relay nodes are selected has great impact on the system performance. It is an optimization problem for selecting suitable relay nodes. The exhaustive search can solve this problem but the complexity will increase factorially with the network size, i.e., the number of users and the number of relays in the network. In this paper, we formulate both single-objective and multi-objective relay selection problems. For single-objective relay selection problem, only one system objective is considered. A novel quantum bee colony optimization (QBCO) based relay selection scheme is proposed. For multiobjective relay selection problem, two contradictive objectives are considered simultaneously. A novel non-dominated sorting quantum bee colony optimization (NSQBCO) based relay selection scheme is proposed. Simulation results show that the proposed relay selection schemes have the ability to find global optimal solution but have less computational complexity compared with exhaustive search scheme.
\end{abstract}

Keywords: Cooperative Relay Networks; Multi-user Relay Selection; Quantum Bee Colony Optimization; Non-dominated Sorting Quantum Bee Colony Optimization

\section{Introduction}

Relaying is an emerging and effective technology which can overcome the limitation of cell coverage and cell edge users' throughput and improve overall system performance of wireless networks [1-2]. It has been well known that introducing relay nodes in 3GPP Long Term Evolution-Advanced (LTE-A) and the conventional cellular networks can help to enlarge the coverage or increase the cell throughput [3]. Relay nodes (RNs) also play an important role in ad hoc networks and other wireless networks such as wireless sensor networks for improving the spatial diversity order and increasing the network longevity [4].

In order to exploit the advantages of the $\mathrm{RN}$ deployment in the wireless networks, relay selection, power and bandwidth allocation for relay nodes have been investigated respectively in literature, in which relay selection is the key issue of the radio resource management (RRM) in relaying systems [5]. The most common relay selection considers the channel state information (CSI) based on physical distance, path loss or signal to noise ratio (SNR), where they focus on the scenario of single source-destination pair with multiple RNs [6-9], which is referred as single-user relay networks. Since the interference does not exist in such scenario, the criterion of relay selection is straightforward. Recently, there is increasing interest in relay networks with multiple source-destination pairs, referred as multiuser relay networks. Typical multi-user relay networks include ad hoc, sensor and mesh networks. However, relay selection schemes proposed for single-user relay 
networks cannot be extended to multi-user relay networks straightforwardly due to the challenges in the performance evaluation, the competition among users and the increased complexity [10].

In the literature, research efforts on relay selection in multi-user networks are rather limited. In [11], for a multi-user network, a relay grouping algorithm is proposed to maximize the minimum achievable rate among users or the network sum-rate respectively. In [12], relay selection scheme that maximizes the minimum achievable rate among all users is proposed. [13] gives an optimality measure for relay selection in multi-user relay networks, which also targets at maximizing the minimum end-to-end receive signal-to-noise ratio (SNR) of all users. But [11-13] assumes that there is no co-channel interference (CCI) among multiple users. In [5] and [14], the multi-user relay selection problem considering CCI between multiple users is formulated and Gale-Shapley based relay selection schemes are proposed to solve the sum-rate maximizing problem. However, only a sub-optimal solution is obtained. To our knowledge researches of relay selection schemes in the literature only consider one objective optimization. No existing research addresses multiobjective optimization which considers contradictive objectives simultaneously in multi-user relay selection problems.

In this paper, we focus on a multi-user network with multiple candidate relays, which suffers from CCI among multiple users. Both single-objective and multiobjective relay selection problems are formulated. In general, the relay selection problems can be formulated as optimization problems and the optimal solution can be obtained by exhaustive search scheme, but the computational complexity is intolerable, which will increase factorially with the network size. In this paper, the intelligent algorithms are used to solve multi-user relay selection problem, which can obtain a near-to-optimal solution but have less computational complexity compared with that of exhaustive search for optimal solution.

Artificial bee colony (ABC) optimization is an effective swarm intelligence method which is widely researched [15-16]. In this paper, we propose a novel multi-relay selection scheme based on quantum bee colony optimization (QBCO) which combine the $\mathrm{ABC}$ proposed in [15-16] with quantum theory to solve singleobjective optimization problems. QBCO has the advantage of both $\mathrm{ABC}$ and quantum theory, thus has a better performance compared with other relay selection schemes. However, multi-objective optimization problems are very different from the single objective optimization problems. In single objective optimization, the goal is to obtain the best design or decision, which is usually the global minimum or global maximum depending on the optimization problem of minimization or maximization. In multi-objective optimization, however, there does not exist one solution which is best with respect to all objectives. Typically, such problems involve tradeoffs. In a typical multi-objective optimization problem, there exists a set of solutions which are superior to the rest of solutions in the search space when all objectives are considered but are inferior to other solutions in the space in one or more objectives. The solutions are known as Pareto front solutions or nondominated solutions. The rest of the solutions are known as dominated solutions. A number of multi objective evolutionary algorithms have been proposed in literature, such as classical NSGA [17], NSGA II [18], SPEA [19] and SPEA2 [20-21] proposes a hybrid evolutionary multi-objective optimization framework. These algorithms are shown to be efficient in the field of multi-criteria optimization and many researchers have investigated their use in different applications. In order to solve multi-objective relay selection problem, we apply the concept of nondominated sorting proposed in NSGA II [18] and propose a novel NSQBCO scheme. To our knowledge, no existing research applies QBCO algorithm in relay selection 
problem of multi-user relay networks and no existing paper addresses NSQBCO in multi-objective relay selection problems.

The rest of the paper is organized as follows. Section 2 describes the network model and problem statement. Section 3 proposes the single-objective multi-user relay selection scheme based on QBCO algorithm. Section 4 proposes the multiobjective multi-user relay selection scheme based on NSQBCO. Section 5 gives the simulation results and Section 6 is the conclusions.

\section{Network Model and Problem Statement}

In this paper, a cooperative multi-user relaying system model is considered, which consists of multiple users for transmission and reception and a certain number of idle nodes which serve as potential relays for cooperation. Each user is referred to as a source node and destination node (SN-DN) transmission pair. $N$ SNs have information to transmit to its own DN, thus formulating $N$ users. Other $M$ idle nodes are potential RNs. Usually $M$ is larger than $N$ [13]. Each user can select one RN to help transmitting. Each RN can help at most one user. There is only one available channel. A two-step decode-and-forward (DF) protocol is used to send information. Two time slots (TSs) are available, i.e., the SNs transmit in TS1 and the RNs transmit in TS2. The RNs can receive in TS1, while the DNs can combine the signals received from SNs and RNs in TS2. Maximum ratio combining (MRC) is used to combine the signals received from SNs and RNs. The transmissions from SNs and RNs are separated into two TSs, so the interference between SNs and RNs is avoided. However, as depicted in Figure 1, the simultaneous transmissions in TS1 from different SNs cause CCI to each other and also the simultaneous transmissions in TS2 from different RNs cause CCI to each other, since there is only one available channel. Scheduling and power control is not considered in this paper. Without loss of generality, the transmission power is assumed to be constant. We also assume there is a centre controller which has all CSI and makes decisions on relay selection then broadcasts the relay assignments on a predefined channel to the SNs, RNs and DNs.

In TS1, the CSI from the $i$-th SN to the $j$-th RN is denoted as $G_{s_{i}, r_{j}}$ and the CSI from the $i$-th SN to the $j$-th DN is denoted as $G_{s_{i}, d_{j}}$. In TS2, the CSI from the $i$-th RN to the $j$-th DN is denoted as $G_{r_{i}, d_{j}}$. For each transmission, the power used at the $i$-th $\mathrm{SN}$ and the $j$-th $\mathrm{RN}$ are $P_{S_{i}}$ and $P_{r_{j}}$, respectively. The power of additive white Gaussian noise (AWGN) is $\eta$ for all links. A node cannot save its power to favor transmissions with better channel realizations. When there is a transmission task between SN and DN, RN either cooperates with its full power or does not cooperate at all. 


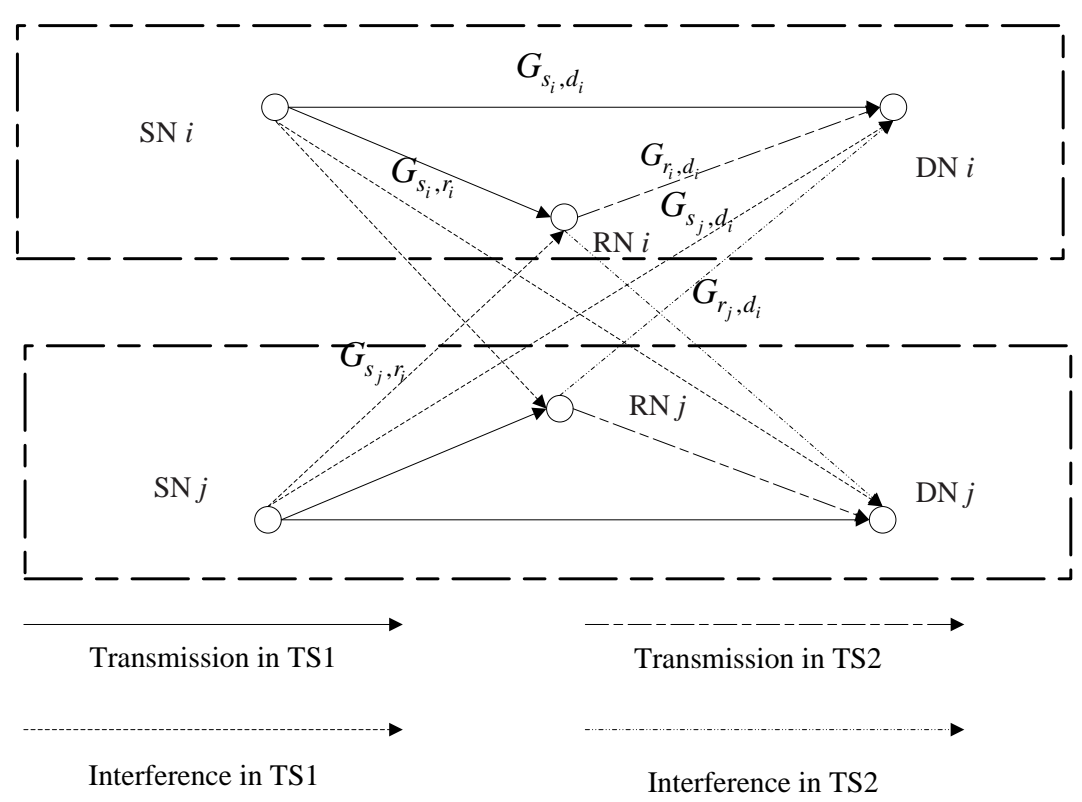

Figure 1. System Model of Multi-User Wireless Relay Network

In TS1, the $i$-th $\mathrm{SN}$ sends $\sqrt{P_{s_{i}}} x_{i}$, where the information symbol is $x_{i}$. If $x_{i}$ is normalized as $E\left|x_{i}\right|^{2}=1$, the average power used at the $i$-th $\mathrm{SN}$ is $P_{s_{i}}$.

The signal received at the $i$-th $\mathrm{DN}$ is

$y_{i}^{(s d)}=\sqrt{G_{s_{i}, d_{i}}} \sqrt{P_{s_{i}}} x_{i}+\sum_{j=1, j \neq i}^{N} \sqrt{G_{s_{j}, d_{i}}} \sqrt{P_{s_{j}}} x_{j}+w \quad$,

Therefore, the signal to interference plus noise ratio (SINR) of SN-DN link can be calculated by

$\gamma_{i}^{(s d)}=G_{s_{i}, d_{i}} P_{s_{i}} /\left(\sum_{j=1, j \neq i}^{N} G_{s_{j}, d_{i}} P_{s_{j}}+\eta\right)$.

The signal received at the $i$-th RN in TS1 can be written as

$y_{i}^{(s r)}=\sqrt{G_{s_{i}, r_{i}}} \sqrt{P_{s_{i}}} x_{i}+\sum_{j=1, j \neq i}^{N} \sqrt{G_{s_{j}, r_{i}}} \sqrt{P_{s_{j}}} x_{j}+w$.

Therefore, the SINR of SN-RN link can be given by

$\gamma_{i}^{(s r)}=G_{s_{i}, r_{i}} P_{s_{i}} /\left(\sum_{j=1, j \neq i}^{N} G_{s_{j}, r_{i}} P_{s_{j}}+\eta\right)$.

$\mathrm{RN}$ receives information in TS1 and decodes the information, then recodes the decoded information and sends the recoded information to the DN, so the signal received at the $i$-th DN is

$y_{i}^{(r d)}=\sqrt{G_{r_{i}, d_{i}}} \sqrt{P_{r_{i}}} x_{i}^{\prime}+\sum_{j=1, j \neq i}^{N} \sqrt{G_{r_{j}, d_{i}}} \sqrt{P_{r_{j}}} x_{j}^{\prime}+w \quad$, 
where $x_{i}^{\prime}$ is the recoded symbol of $x_{i}$.

Therefore, the SINR of RN-DN link can be expressed as

$\gamma_{i}^{(r d)}=G_{r_{i}, d_{i}} P_{r_{i}} /\left(\sum_{j=1, j \neq i}^{N} G_{r_{j}, d_{i}} P_{r_{j}}+\eta\right)$

For DF relaying, the achievable data rate under the two-time-slot structure given by $[12-22]$ is

$$
\begin{aligned}
& R_{i}=\frac{1}{2} W \log _{2}\left(1+\gamma_{i}\right)=\frac{1}{2} W \log _{2}\left(1+\min \left\{\gamma_{i}^{(s r)}, \gamma_{i}^{(s d)}+\gamma_{i}^{(r d)}\right\}\right), \\
& =\frac{1}{2} W \min \left\{\log _{2}\left(1+\gamma_{i}^{(s r)}\right), \log _{2}\left(1+\gamma_{i}^{(s d)}+\gamma_{i}^{(r d)}\right)\right\}
\end{aligned},
$$

where $W$ is the bandwidth of the available channel, $\gamma_{i}$ is the end-to-end SINR of user $i$. Three single-objective relay selection problems are formulated:

1) Max-Average-Reward (MAR): This maximizes the average throughput of network regardless of fairness. The optimization problem is expressed as

$$
\max _{\mathbf{R}} \mathrm{U}_{\mathrm{MAR}}(\mathbf{R}) \square \frac{1}{N} \sum_{i=1}^{N} R_{i} \text {. }
$$

2) Max-Proportional-Fair (MPF): This maximizes the fairness of throughput between different users of the network. The optimization problem is expressed as

$\max _{\mathbf{R}} \mathrm{U}_{\mathrm{MPF}}(\mathbf{R}) \square\left(\prod_{i=1}^{N} R_{i}\right)^{\frac{1}{N}}$.

3) Max-Min-Reward (MMR): This maximizes the throughput of the bottleneck user, which also considers fairness. The optimization problem is expressed as

$\max _{\mathbf{R}} \mathrm{U}_{\mathrm{MMR}}(\mathbf{R}) \square \min _{1 \leq i \leq N}\left\{R_{i}\right\}$

where $\mathbf{R}=\left[r_{1}, r_{2}, \cdots, r_{N}\right]$ is the relay selection scheme, and if $i \neq j$ then $r_{i} \neq r_{j}$ $(i, j=1,2, \cdots, N)$, which guarantees that each $\mathrm{RN}$ can help at most one user. Each element $r_{i}(i=1,2, \cdots, N)$ denotes the $\mathrm{RN}$ selected by user $i$, so if $\mathrm{RN} k(k=1,2, \cdots, M)$ is assigned to user $i$, then $r_{i}=k$.

Since the MAR only considers the average throughput of network, the relay selection scheme which has the largest MAR value cannot obtain the largest MPF or MMR value. Also, the relay selection which has the largest MMR value cannot obtain the largest MPF. Considering two objectives simultaneously, three multi-objective relay selection problems are formulated, which are in the following:

$$
\max _{\mathbf{R}}\left\{\begin{array}{l}
\mathrm{U}_{\mathrm{MAR}}(\mathbf{R}) \square \frac{1}{N} \sum_{i=1}^{N} R_{i} \\
\mathrm{U}_{\mathrm{MPF}}(\mathbf{R}) \square\left(\prod_{i=1}^{N} R_{i}\right)^{\frac{1}{N}},
\end{array}\right.
$$




$$
\begin{gathered}
\max _{\mathbf{R}}\left\{\begin{array}{l}
\mathrm{U}_{\mathrm{MAR}}(\mathbf{R}) \square \frac{1}{N} \sum_{i=1}^{N} R_{i} \\
\mathrm{U}_{\mathrm{MMR}}(\mathbf{R}) \square \min _{1 \leq i \leq N}\left\{R_{i}\right\}
\end{array},\right. \\
\max _{\mathbf{R}}\left\{\begin{array}{l}
\mathrm{U}_{\mathrm{MMR}}(\mathbf{R}) \square \min _{1 \leq i \leq N}\left\{R_{i}\right\} \\
\mathrm{U}_{\mathrm{MPF}}(\mathbf{R}) \square\left(\prod_{i=1}^{N} R_{i}\right)^{\frac{1}{N}} .
\end{array}\right.
\end{gathered}
$$

For each relay selection scheme $\mathbf{R}$, the CCI makes the optimization problem too complicated to solve. Exhaustive search to obtain the solution would involve the following procedures. For all possible solutions, compute all SINRs and the achievable data rate. Hence, all $M ! /(M-N)$ ! (the number of ordered sequences of $N$ elements selected from a set of $M$ elements) possible relay selection schemes must be evaluated and compared. Since factorial function grows faster than exponential function, the complexity of the exhaustive search is intolerable for realistic values of $M$ and $N$, especially when $M$ is large. In this paper, the novel intelligent algorithms, QBCO and NSQBCO, are used for solving single-objective and multi-objective relay selection problems respectively.

\section{Single-Objective Relay Selection Scheme}

Since relay selection problem proposed in Section 2 is an integer optimization problem, we propose QBCO based scheme to solve relay selection problem in multi-user cooperative relay networks.

QBCO is a novel multi-agent optimization system modified by ABC [15-16]. It is inspired by social behavior of bees. The colony of quantum bees including three groups of bees: quantum employed bees, quantum onlooker bees and quantum scouts bees. They look for food resources (which are represented by quantum position) in an $\mathrm{N}$-dimensional space according to the historical experiences of its own and its colleagues'; where $N$ represents the dimension of the optimization problem $(N$ represents the number of SNDN transmission pairs in the multi-user relay selection problem). The quantum position of the $h$-th $(h=1,2, \cdots, H)$ quantum bee is defined as

$$
\mathbf{x}_{h}=\left[\begin{array}{cccc}
\alpha_{h 1} & \alpha_{h 2} & \cdots & \alpha_{h N} \\
\beta_{h 1} & \beta_{h 2} & \cdots & \beta_{h N}
\end{array}\right]
$$

where $H$ is the number of food resources, $\left|\alpha_{h i}\right|^{2}+\left|\beta_{h i}\right|^{2}=1, \quad(i=1,2, \cdots, N)$. For simplicity and efficient design of QBCO, we define $\alpha_{h i}$ and $\beta_{h i}$ as real numbers and $0 \leq \alpha_{h i} \leq 1,0 \leq \beta_{h i} \leq 1$. Therefore, $\alpha_{h i}=\sqrt{1-\beta_{h i}^{2}}$ and (14) can be simplified as

$\mathbf{x}_{h}=\left[\begin{array}{llll}\alpha_{h 1} & \alpha_{h 2} & \cdots & \alpha_{h N}\end{array}\right]=\left[\begin{array}{llll}x_{h 1} & x_{h 2} & \cdots & x_{h N}\end{array}\right]$

\subsection{Evolutionary Process of Quantum Employed Bees}

The evolutionary process of quantum employed bees' quantum position is mainly computed through quantum rotation gate. In our scheme, for simplicity, the $i$-th quantum position $x_{h i}$ is updated as 


$$
x_{h i}^{t+1}=\left|x_{h i}^{t} \times \cos \theta_{h i}^{t+1}-\sqrt{1-\left(x_{h i}^{t}\right)^{2}} \times \sin \theta_{h i}^{t+1}\right|
$$

where superscript $t$ and $t+1$ represent number of iterations (generations), $|\cdot|$ is an absolute function which makes quantum position in the real domain $[0,1]$, and $\theta_{h i}^{t+1}$ is the quantum rotation angle, which can be calculated through (18).

If $\theta_{h i}^{t+1}=0$, the quantum position $x_{h i}$ is updated by the operator described as $x_{h i}^{t+1}=\sqrt{1-\left(x_{h i}^{t}\right)^{2}}$.

After the $t$-th generation, the best quantum position (the local optimal quantum position) of the $h$-th quantum bee is $\mathbf{p}_{h}^{t}=\left[\begin{array}{llll}p_{h 1}^{t} & p_{h 2}^{t} & \cdots & p_{h N}^{t}\end{array}\right]$, and the global optimal quantum position discovered by the whole quantum bee population is $\mathbf{p}_{g}^{t}=\left[\begin{array}{llll}p_{g 1}^{t} & p_{g 2}^{t} & \cdots & p_{g N}^{t}\end{array}\right]$. At each generation, the $h$-th quantum bee's quantum rotation angles and quantum positions are updated by the following quantum moving equations:

$\theta_{h i}^{t+1}=\frac{1}{2} \cdot \xi_{1} \cdot\left(p_{h i}^{t}-x_{h i}^{t}\right)+\frac{1}{2} \cdot \xi_{2} \cdot\left(p_{g i}^{t}-x_{h i}^{t}\right)$

$x_{h i}^{t+1}=\left\{\begin{array}{l}\sqrt{1-\left(x_{h i}^{t}\right)^{2}}, \quad p_{h i}^{t}=x_{h i}^{t}=p_{g i}^{t} \\ \operatorname{abs}\left(x_{h i}^{t} \times \cos \theta_{h i}^{t+1}-\sqrt{1-\left(x_{h i}^{t}\right)^{2}} \times \sin \theta_{h i}^{t+1}\right), \quad \text { otherwise }\end{array}\right.$

where $\xi_{1}$ and $\xi_{2}$ are Gaussian distributed random numbers with zero mean and unit variance. The values of $\xi_{1}$ and $\xi_{2}$ express the relative important degree of $\mathbf{p}_{h}^{t}$ and $\mathbf{p}_{g}^{t}$.

After updating the quantum position of each quantum employed bee, the quantum position is mapped into continuous number, and the rule can be described as follows

$\overline{x_{h i}}=l_{i}+x_{h i}\left(u_{i}-l_{i}\right)$

where $l_{i}$ is the lower bound of the $i$-th dimension instant, $u_{i}$ is the upper bound of the $i$ th dimension instant. In the multi-user relay selection scheme, there are $M$ potential relays which can be chosen, so $l_{i}=1, u_{i}=M$ for all $i=1,2, \cdots, N$.

Since the multi-user relay selection problem is an integer optimization problem, we should map the continuous number into different integers, the rule is

$\overline{\overline{x_{h i}}}=F\left(\overline{x_{h i}}\right)$,

where $F(x)$ means the integer nearest to $x$.

Then compute the fitness of each quantum bee according to reward function (8-10). In this step, penalty factor is used to delete the infeasible solutions. That is to say, if one solution does not satisfy the condition that each RN can help at most one user, which can be written as $\overline{\overline{x_{h i}}} \neq \overline{\overline{x_{h j}}}(\forall i \neq j, i, j=1,2, \cdots, N)$, the fitness of the solution is set to a negative value. At last, the local optimal and global optimal solutions are updated. If the fitness of $\mathbf{x}_{h}^{t+1}$ is better than that of $\mathbf{p}_{h}^{t}, \mathbf{p}_{h}^{t+1}$ is updated as $\mathbf{x}_{h}^{t+1}$. If the fitness of $\mathbf{p}_{h}^{t+1}$ is better than that of $\mathbf{p}_{g}^{t}, \mathbf{p}_{g}^{t+1}$ is updated as $\mathbf{p}_{h}^{t+1}$. 


\subsection{Evolutionary Process of Quantum Onlooker Bees}

The evolutionary process of quantum onlooker bees' quantum position is based on the selected quantum bee's quantum position, which guide the evolutionary process of quantum onlooker bee.

The selection possibility of the $k$-th quantum employed bee can be defined as follows:

$$
p_{k}=\frac{\mathrm{U}\left(\overline{\overline{\mathbf{x}_{k}}}\right)}{\sum_{i=1}^{H} \mathrm{U}\left(\overline{\overline{\mathbf{x}_{i}}}\right)}
$$

where $\overline{\overline{\mathbf{x}_{k}}}$ is obtained according to (20) and (21) by $\mathbf{x}_{k}$.

At each generation, the $h$-th quantum onlooker bee's quantum rotation angles and quantum positions are updated by the following quantum moving equations, assume that the $k$-th quantum employed bee is selected as the guidance of the quantum onlooker bee:

$$
\begin{aligned}
& \theta_{h i}^{t+1}=\frac{1}{2} \cdot \xi_{1} \cdot\left(p_{k i}^{t}-x_{h i}^{t}\right)+\frac{1}{2} \cdot \xi_{2} \cdot\left(p_{g i}^{t}-x_{h i}^{t}\right) \\
& x_{h i}^{t+1}=\left\{\begin{array}{l}
\sqrt{1-\left(x_{h i}^{t}\right)^{2}}, \quad p_{h i}^{t}=x_{h i}^{t}=p_{g i}^{t} \\
\operatorname{abs}\left(x_{h i}^{t} \times \cos \theta_{h i}^{t+1}-\sqrt{1-\left(x_{h i}^{t}\right)^{2}} \times \sin \theta_{h i}^{t+1}\right), \text { otherwise }
\end{array}\right.
\end{aligned}
$$

where $\xi_{1}$ and $\xi_{2}$ are Gaussian distributed random numbers with zero mean and unit variance. The values of $\xi_{1}$ and $\xi_{2}$ express the relative important degree of $\mathbf{p}_{h}^{t}$ and $\mathbf{p}_{g}^{t}$.

After updating the quantum position of each quantum onlooker bee, the fitness is computed as the process of quantum employed bee. Also, the local optimal and global optimal solutions are updated as the process of quantum employed bee.

\subsection{Evolutionary Process of Quantum Onlooker Bees}

When the fitness of each quantum employed bee or quantum onlooker bee does not change in limit times, then it becomes a quantum scout bee, which has the ability to find new quantum position, thus the quantum position is selected randomly. The fitness of quantum scout bee is computed as the process of quantum employed bee. Also, the local optimal and global optimal solutions are updated as the process of quantum employed bee.

\subsection{The Process of QBCO Based Relay Selection Scheme}

Based on what we have discussed, QBCO can be applied to solve multi-user relay selection problem in cooperative relay networks. The process of QBCO based relay selection scheme is shown below:

Step1: Assume that the centre controller knows all CSI and the centre controller completes the relay selection process.

Step2: Randomly generate an initial quantum bee colony based on quantum coding mechanism.

Step3: According to the evolutionary process of quantum employed bee, quantum onlooker bee and quantum scout bee, perform the evolution scheme, calculate the fitness and renew each quantum bee's local optimal position. 
Step4: Update the global optimal position as evolutionary objective of the whole quantum bee colony.

Step5: If it reaches the predefined value of the maximum generation, stop the process, and then transfer the outcome to the relay selection scheme $\mathbf{R}$ according to (21); if not, then go to Step 3.

Step6: The centre controller broadcasts the relay assignments on a predefined channel to the SNs, RNs and DNs. The relay selection process is end.

\section{Multi-Objective Relay Selection Scheme}

Considering two objectives simultaneously, i.e., MAR and MPF (11), or MAR and MMR (12), or MMR and MPF (13), we propose the NSQBCO scheme to solve the multi-objective problems thus obtain Pareto solutions. The NSQBCO is based on non-dominated sorting and crowding distance [18], where the entire population is sorted into various non-dominated levels. This provides the means for selecting the solutions in the better fronts, hence providing the necessary selection pressure to push the population towards the Pareto front.

\subsection{Non-Dominated Sorting and Crowding Distance}

First, we explain the definition of non-dominated solutions. If we want to maximize $f_{j}(\mathbf{x}) \quad(j=1, \cdots, J)$, where $J$ is the number of objectives we want to optimize, then for solutions $\mathbf{u}$ and $\mathbf{v}$, if $\forall j=1, \cdots, J, f_{j}(\mathbf{u}) \geq f_{j}(\mathbf{v})$, and $\exists j=1, \cdots, J, f_{j}(\mathbf{u})>f_{j}(\mathbf{v})$ then define $\mathbf{u}$ dominates $\mathbf{v}$, and $\mathbf{u}$ is a non-dominated solution or Pareto front solution. This means for all objectives, solution $\mathbf{u}$ is not worse than solution $\mathbf{v}$ and at least there exists one objective which solution $\mathbf{u}$ is better than solution $\mathbf{v}$.

Then the process of non-dominated sorting can be described as follows: 1) For each solution calculate two entities: domination count $n_{\mathbf{x}}$ (which is the number of solutions that dominate $\mathbf{x}$ ) and $\mathbf{S}_{\mathbf{x}}$ (which contains all the solutions that are being dominated by $\mathbf{x}$ ). 2) All solutions in the first non-dominated front will have their domination count as zero. Now, for each solution $\mathbf{x}$ with $n_{\mathbf{x}}=0$, visit each member $\mathbf{y}$ of its set $\mathbf{S}_{\mathbf{x}}$ and reduce its domination count by one. In doing so, if for any member $\mathbf{y}$ the domination count becomes zero, it is put in a separate list $\mathbf{Q}$. These members belong to the second nondominated front. Now the above procedure is continued with each member of $\mathbf{Q}$ and the third front is identified. This process continues until all fronts are identified. Solutions in the first non-dominated front are the non-dominated solutions among all solutions.

Along with convergence to the Pareto front, it is also desired that the algorithm maintains a good spread of solutions in the obtained set of solutions. We calculate the average distance of two points along each of the objectives. The crowding distance is used to maintain population diversity, and the calculation process will be described in the following.

The crowding-distance computation requires sorting the population according to each objective value in ascending order of magnitude for every front. Therefore, for each objective function, the boundary solutions (solutions with smallest and largest function values) are assigned an infinite distance value. All other intermediate solutions are assigned a distance value equal to the absolute normalized difference in the function values of two adjacent solutions. The calculation is continued with other objective functions. The overall crowding distance value is calculated as the sum of individual distance values corresponding to each objective.

From the description of non-dominated sorting and crowding distance, we can see that the solutions with better front and larger crowding distance are better than others. 


\subsection{Non-Dominated Sorting Quantum Bee Colony Optimization}

The NSQBCO uses QBCO proposed in Section 3 as the evolutionary scheme. The process can be summarized in the following steps:

Step 1: Initialize quantum bee colony $\mathbf{S}$ based on quantum coding mechanism. The number of quantum bees in $\mathbf{S}$ is recorded as $H$.

Step 2: Evaluate each quantum bee (i.e., calculate the value of different objectives) and sort population $\mathbf{S}$ according to non-dominated sorting scheme.

Step 3: Generate new quantum bee colony $\mathbf{S}_{\text {employed }}$ through evolutionary scheme of employed bee based on QBCO. Both the global best solution $\mathbf{p}_{g}$ and the local best solution of the $h$-th quantum bee $\mathbf{p}_{h}(h=1,2, \cdots, H / 2)$ are chosen from $\mathbf{S}$ randomly.

Step 4: Generate new quantum bee colony $\mathbf{S}_{\text {onlooker }}$ through evolutionary scheme of onlooker bee based on QBCO. The global best solution $\mathbf{p}_{g}$ is chosen from $\mathbf{S}$ randomly and solution of the selected quantum employed bee $\mathbf{p}_{k}(k=H / 2+1, H / 2+2, \cdots, H)$ are chosen from $\mathbf{S}_{\text {employed }}$ randomly.

Step 5: Combine $\mathbf{S}$ with $\mathbf{S}_{\text {employed }}$ and $\mathbf{S}_{\text {onlooker }}$ thus form a new quantum bee colony $\mathbf{S}_{\text {new }}$. Evaluate each quantum bee (i.e., calculate the value of different objectives). If the solution is not updated in limit times, then it becomes a quantum scout bee, update the relative solution. Sort $\mathbf{S}_{\text {new }}$ according to non-dominated sorting scheme.

Step 6: Update the individuals that will take part in the next iteration, i.e., replace $\mathbf{S}$ with the best $H$ quantum bee in $\mathbf{S}_{\text {new }}$.

Step7: If it has reached the maximum generation, then stop the process. The nondominated solutions in $\mathbf{S}$ are non-dominated solutions. Otherwise, go to Step 3 until it has reached the maximum generation.

From the above process, in each iteration, we select the non-dominated solutions in the current population and reject the dominated solutions. Through the iteration of evolutionary process, we can obtain the Pareto front solutions.

\subsection{NSQBCO Based Muti-Objective Relay Selection Scheme}

According to the above analysis, the process of NSQBCO based multi-objective relay selection scheme is shown below:

Step1: Assume that the centre controller knows all CSI and the centre controller completes the relay selection process.

Step2: Using NSQBCO scheme (while one objective is MAR and the other MPF or one objective is MAR and the other is MMR or one objective is MMR and the other is MPF) to obtain the Pareto front solutions.

Step3: The centre controller chooses one solution from the Pareto solutions according to the tradeoff of MAR and MPF or the tradeoff of MAR and MMR or the tradeoff of MMR and MPF and broadcasts the relay assignments on a predefined channel to the SNs, RNs and DNs. The relay selection process is ended.

\section{Simulation Results and Analysis}

In this section, firstly we present the performance of single-objective relay selection scheme, including the proposed QBCO based single-objective relay selection scheme, $\mathrm{ABC}$ based relay selection scheme and Gale-Shapley based relay selection schemes [514]. The process of ABC based relay selection scheme is similar to QBCO based relay selection scheme except for the bees' position and velocity updating process. For more 
details of $\mathrm{ABC}$ algorithm, please refer to [15-16]. Gale-Shapley based relay selection schemes are also presented for comparison, which include Gale-Shapley-Min scheme and Gale-Shapley-Harmonic scheme. For more details of Gale-Shapley based schemes, please refer to [5-14].

In the simulations, the bandwidth is set to be $10 \mathrm{MHz}$ for the available channel [12], i.e., $W=10 \mathrm{MHz}$. The path gain $G_{i, j}$ between two nodes is modeled as $G_{i, j}=d_{i, j}^{-3}$, where $d_{i, j}$ is the distance of the two nodes. Wireless links and nodes are uniformly distributed over a square field with dimension $D \times D$, in the simulation process, set $D=100$. In each simulation, $N$ SNs and DNs are randomly generated while guaranteeing the distance between $\mathrm{SN}$ and $\mathrm{DN}$ is uniformly distributed between $\left[d_{\min }, d_{\max }\right]$, so that they are not too far away from each other, in the simulation, $d_{\min }=25, d_{\max }=35$. Then $M$ candidate relays are generated in the area. The power of different SNs is the same, as well as the power of RNs. The power of AWGN is $10^{-3} \mathrm{~W}$ at all nodes, i.e., $\eta=10^{-3} \mathrm{~W}$. For QBCO scheme and $\mathrm{ABC}$ scheme, the maximal iteration is 500 and $H=20$. For $\mathrm{ABC}$ scheme, the parameter settings are referred to [15-16]. All results are averaged over 1000 cases.

Compare the proposed scheme with exhaustive search, ABC scheme and GaleShapley based schemes when $N=5, M=7$ (if $N, M$ is large, the computer is out of memory when calculating all possible solutions and choose the best one, i.e., exhaustive search) as the power of $\mathrm{SN}$ varies from $5 \mathrm{~W}$ to $40 \mathrm{~W}$. The RN power is $10 \mathrm{~W}$. Simulation results are shown in Figure 2. The QBCO scheme performs better than ABC scheme and Gale-Shapley based schemes. The gap between QBCO scheme and Gale-Shapley schemes is more than $0.3 \mathrm{Mbit} / \mathrm{s}$, especially when the $\mathrm{SN}$ power is larger, the gap is more than $0.5 \mathrm{Mbit} / \mathrm{s}$. It obtains almost the same performance as exhaustive search, which verifies the effectiveness of QBCO scheme.

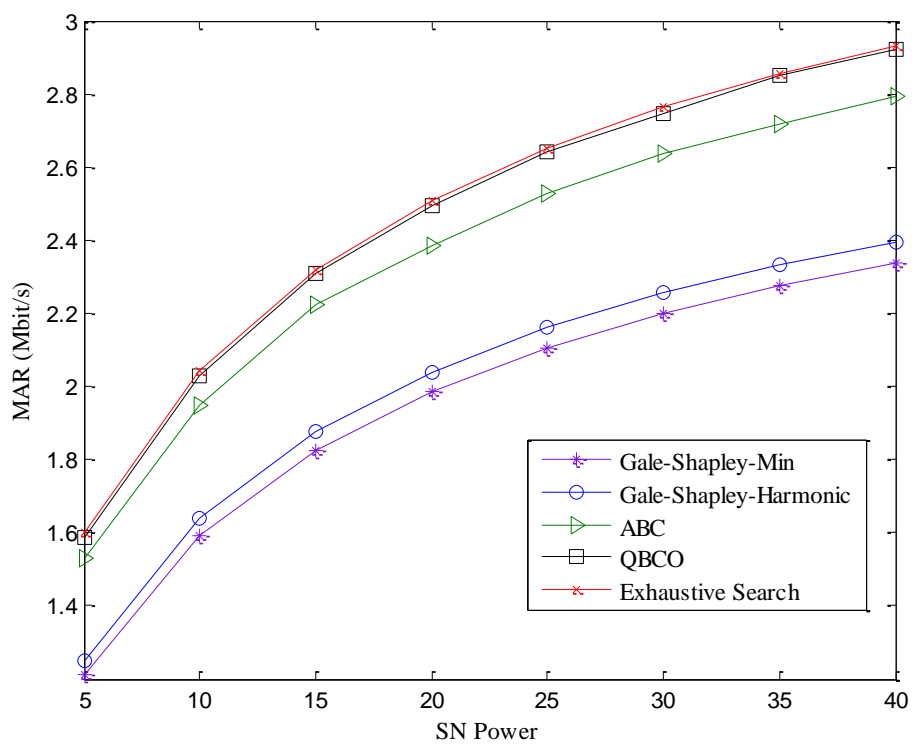

Figure 2. The Comparison of MAR for QBCO scheme, ABC Scheme, GaleShapley Schemes and Exhaustive Search with SN Power

Figure 3 considers the case where the MAR, MPF and MMR varies with the RN number when $N=10$, the $\mathrm{SN}$ power is $20 \mathrm{~W}$ and the $\mathrm{RN}$ power is $10 \mathrm{~W}$. From Figure 3 , it is obvious that the MAR, MPF and MMR increase with the RN number. This is easy to understand, with the increasing number of candidate relays, each user has a higher 
probability to select a 'better' relay, thus enhancing the performance. Also, QBCO scheme performs better than ABC scheme for MAR, MPF and MMR target, especially for MPF target. This means that our scheme, QBCO scheme, has an excellent performance under different simulation conditions.

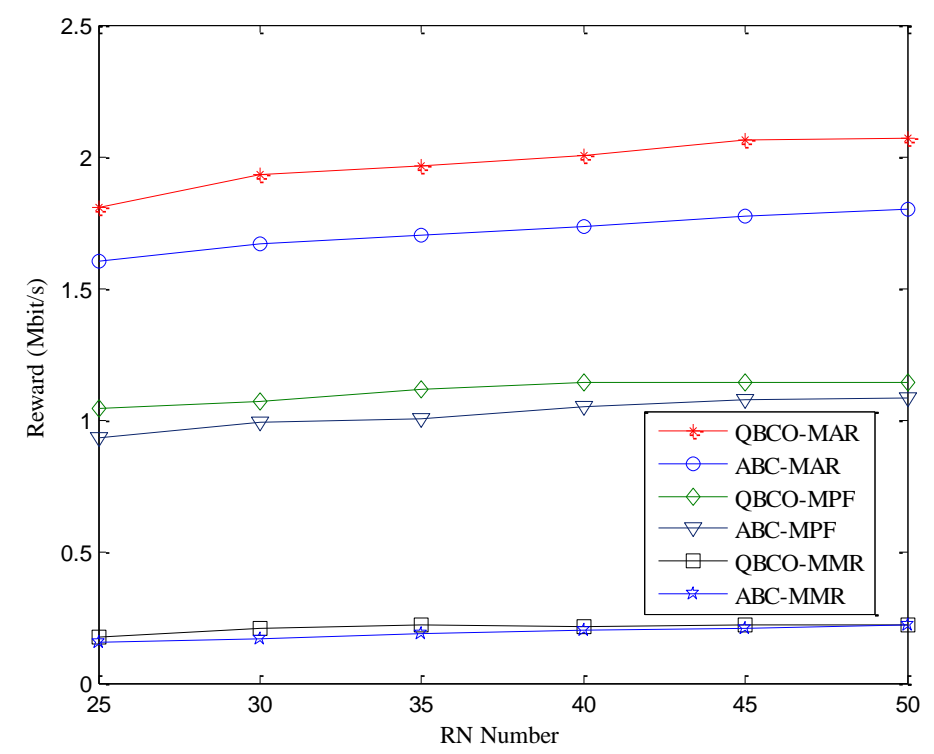

Figure 3. The Comparison of MAR, MPF and MMR for QBCO Scheme, ABC Scheme with RN Number

Next, we focus on the performance of multi-objective relay selection scheme. For NSQBCO scheme, the maximal iteration is 200 and $H=50$. Figure 4-6 present the performance of all possible solutions referred to as exhaustive search and the solutions obtained by NSQBCO when $N=5, M=7$. The power of $\mathrm{SN}$ is $20 \mathrm{~W}$ while the RN power is $10 \mathrm{~W}$.

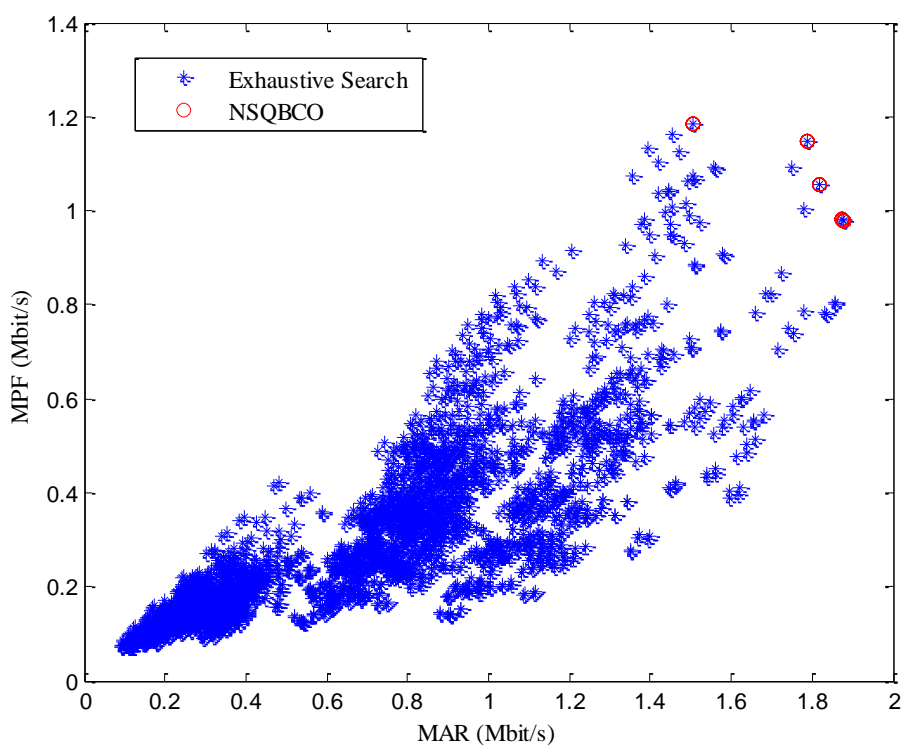

Figure 4. All Solutions in One Relay Selcetion Case and Solutions Obtained by NSQBCO Considering MAR and MPF 


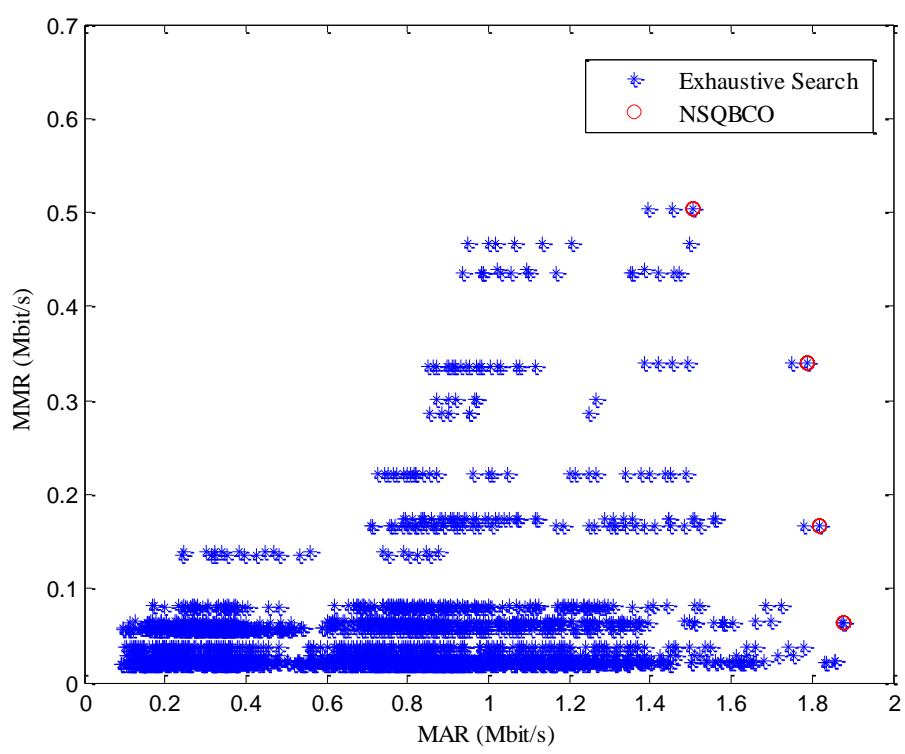

Figure 5. All Solutions in One Relay Selcetion Case and Solutions Obtained by NSQBCO Considering MAR and MMR

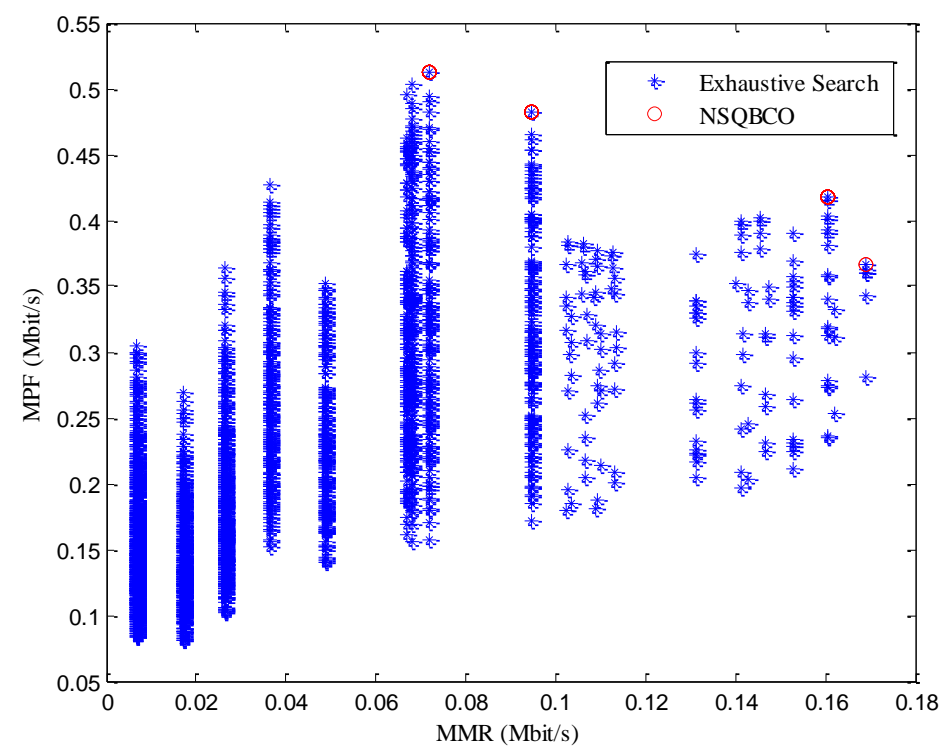

Figure 6. All Solutions in One Relay Selcetion Case and Solutions Obtained by NSQBCO Considering MMR and MPF

Figure 4 considers MAR and MPF, and it can be seen there does not exist one solution which maximizes MAR and MPF simultaneously. If one solution has the largest MAR value, the performance of MPF is poor. Also if one solution has the largest MPF value, the MAR is limited, which demonstrates that MAR and MPF objectives are contradictive. Figure 5 considers MAR and MMR, Figure 6 considers MMR and MPF, which has the same phenomenon. Also, the solutions obtained by NSQBCO are all Pareto front solutions, i.e., there does not exist one solution that performs better in both objectives compared with these solutions. This demonstrates the effectiveness of the NSQBCO scheme. 
When the network size becomes larger, exhaustive search cannot be used due to algorithm complexity. But the NSQBCO can still be used to solve multi-objective relay selection problems. The solutions are presented in Figure 7 when $N=10, M=20$, the power of $\mathrm{SN}$ is $20 \mathrm{~W}$ while the $\mathrm{RN}$ power is $10 \mathrm{~W}$. The solutions obtained by QBCO scheme for MAR and MMR target are also presented for comparison. The solutions obtained by QBCO scheme incorporate the solutions obtained by NSQBCO scheme. This demonstrates that the multi-objective scheme has a wider application field compared with single-objective schemes. All these present the advantage of the proposed singleobjective QBCO based and multi-objective NSQBCO based relay selection schemes.

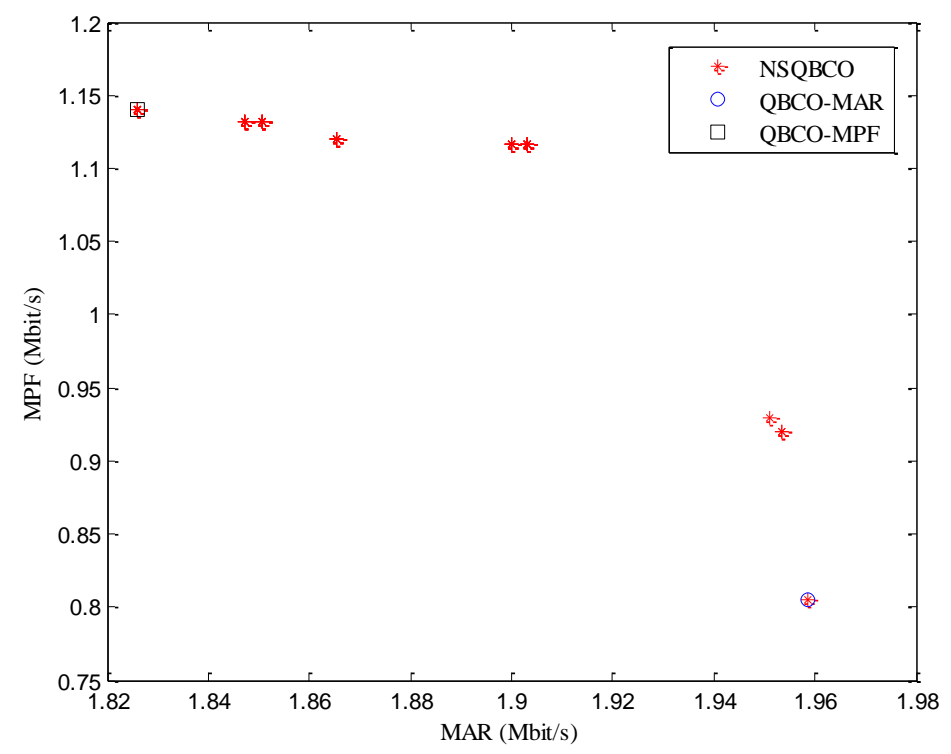

\section{Figure 7. The Performance of NSQBCO Scheme with Larger Network Size Considering MAR and MPF}

The solutions for MAR and MMR optimization are presented in Figure 8 when $N=10, M=20$, the power of $\mathrm{SN}$ is $20 \mathrm{~W}$ while the $\mathrm{RN}$ power is $10 \mathrm{~W}$. The solutions obtained by QBCO scheme for MAR and MMR target are also presented for comparison. The solutions obtained by QBCO scheme incorporate the solutions obtained by NSQBCO scheme. It also presents that the solution which the largest MAR value, its' MMR performance is rather limited, which demonstrate the significance of multiobjective optimization.

The solutions for MMR and MPF optimization are presented in Figure 9 when $N=10, M=20$, the power of $\mathrm{SN}$ is $20 \mathrm{~W}$ while the $\mathrm{RN}$ power is $10 \mathrm{~W}$. The solutions obtained by QBCO scheme for MMR and MPF target are also presented for comparison. The solutions present the advantage of the proposed single-objective QBCO based and multi-objective NSQBCO based relay selection schemes. 


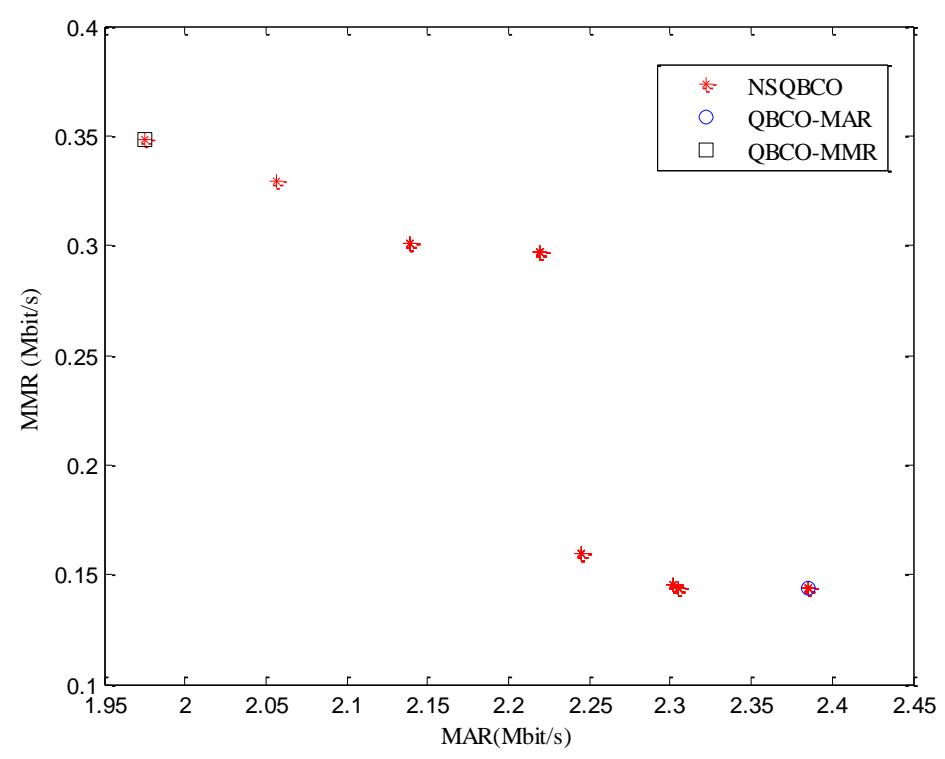

Figure 8. The Performance of NSQBCO Scheme with Larger Network Size Considering MAR and MMR

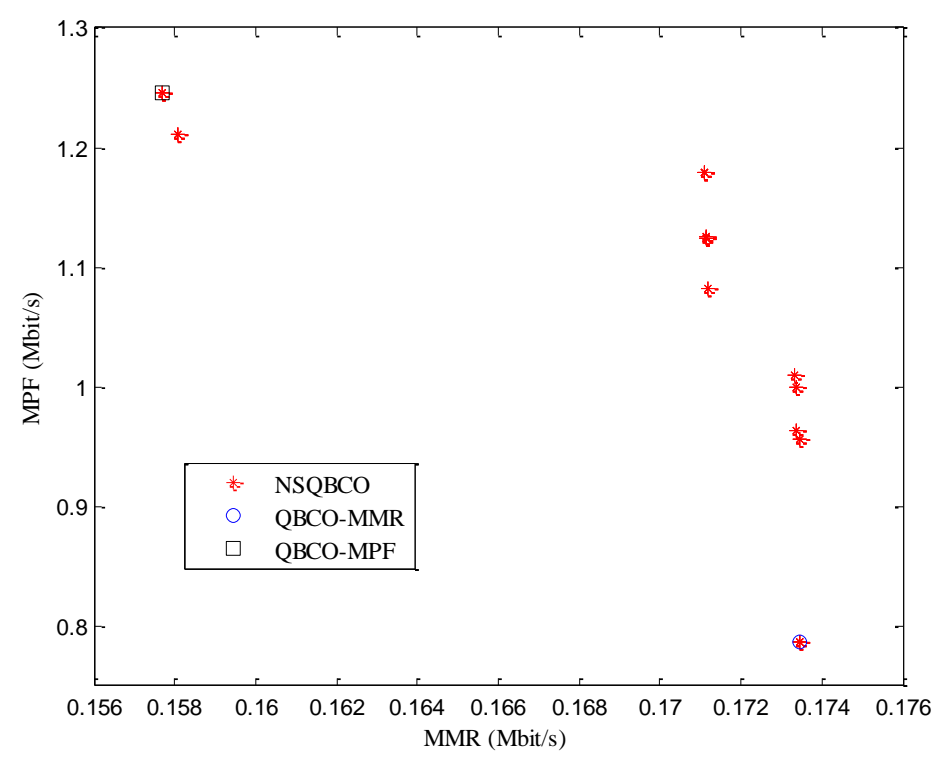

Figure 9. The Performance of NSQBCO Scheme with Larger Network Size Considering MMR and MPF

\section{Conclusions}

This paper has proposed multi-user relay selection schemes considering CCI to other users for both single-objective and multi-objective relay selection problems in the cooperative relay networks. Firstly, QBCO based single-objective relay selection scheme is proposed to solve MAR, MMR and MPF optimization problems. Compared with other single-objective relay selection schemes in the literature, the proposed QBCO scheme has much better performance under different simulation scenarios. Then, NSQBCO based multi-objective relay selection scheme is 
proposed to obtain Pareto front solutions for MAR and MPF optimization, MAR and MMR optimization, MPF and MMR optimization. Simulation results illustrate the effectiveness of NSQBCO scheme, which has a wider application field compared with single-objective schemes, which validate that the multi-objective schemes have some wider applications.

\section{Acknowledgments}

First of all, I want to take this chance to thank my tutor Prof. Ming Diao and senior fellow apprentice Hongyuan Gao, then thank everyone that help and support me without reservation especially my family.

\section{References}

[1] A. Nosratinia, T. E. Hunter and A. Hedayat, "Cooperative communication in wireless networks", Communications Magazine, IEEE, vol. 42, no.10, (2004) October, pp. 74-80

[2] A. V. Babu and S. Joshy, "Maximizing the data transmission rate of a cooperative relay system in an underwater acoustic channel", International Journal of Communication Systems vol. 25, (2012), pp. 231-253.

[3] 3GPP TR 36.814, "Further Advancement for E-UTRA Physical Layer Aspects”, vo1. 5, no. 2, (2009) December.

[4] F. Etezadi, K. Zarifi, A. Ghrayeb and S. Affes, "Decentralized Relay Selection Schemes in Uniformly Distributed Wireless Sensor Networks", Wireless Communications, IEEE Transactions on, vol. 11, no. 3, (2012) March, pp. 938-951.

[5] S. Zhou, J. Xu and Z. Niu, "Interference-Aware Relay Selection Scheme for Two-Hop Relay Networks with Multiple Source-Destination Pairs", Vehicular Technology, IEEE Transactions on, vol. PP, no. 99, (2013), pp. 1-12.

[6] A. Bletsas, A. Khisti, D. P. Reed and A. Lippman, "A simple Cooperative diversity method based on network path selection", Selected Areas in Communications, IEEE Journal on, vol. 24, no. 3, (2006), March, pp. 659-672.

[7] J. Yindi and H. Jafarkhani, "Single and multiple relay selection schemes and their achievable diversity orders", Wireless Communications, IEEE Transactions on, vol. 8, no. 3, (2009) March, pp. 1414-1423.

[8] D. Michalopoulos and G. Karagiannidis, "Performance analysis of single relay selection in rayleigh fading”, Wireless Communications, IEEE Transactions on, vol. 7, no. 10, (2008) October, pp. 37183724.

[9] M. Zhou, Q. Cui, R. Jantti and T. Xiaofeng, "Energy-Efficient Relay Selection and Power Allocation for Two-Way Relay Channel with Analog Network Coding”, Communications Letters, IEEE, vol. 16, no. 6, (2012) June, pp. 816-819.

[10] N. T. C. Yam and Y. Wei, "Joint optimization of relay strategies and resource allocations in cooperative cellular networks", Selected Areas in Communications, IEEE Journal on, vol. 25, no. 2, (2007) February, pp. 328-339.

[11] C. Esli and A. Wittneben, "A Hierarchical AF Protocol for Distributed Orthogonalization in Multiuser Relay Networks, Vehicular Technology”, IEEE Transactions on, vol. 59, no. 8, (2010) October, pp. 3902-3916.

[12] S. Sharma, S. Yi, Y. T. Hou and S. Kompella, "An Optimal Algorithm for Relay Node Assignment in Cooperative Ad Hoc Networks", Networking, IEEE/ACM Transactions on, vol. 19, no. 3, (2011) June, pp. 879-892.

[13] S. Atapattu, Y. Jing, H. Jiang and C. Tellambura, "Relay Selection and Performance Analysis in Multiple-User Networks", Selected Areas in Communications, IEEE Journal on, vol. 31, no. 8, (2013) August, pp. 1-13.

[14] X. Jie, Z. Sheng and N. Zhisheng, "Interference-aware relay selection for multiple source-destination cooperative networks, in Communications", 2009. APCC 2009. 15th Asia-Pacific Conference on, (2009), pp. 338-341.

[15] D. Karaboga and B. Basturk, "On the performance of artificial bee colony (ABC) algorithm, Applied Soft Computing", vol. 8, (2008) June, pp. 687-697.

[16] D. Karaboga and B. Basturk, "A powerful and efficient algorithm for numerical function optimization: artificial bee colony(ABC) algorithm”, Journal of Global Optimization, vol. 39, (2007), pp. 459-471.

[17] N. Srinivas and K. Deb, "Mutiobjective optimization using nondominated sorting in genetic algorithms", Evolutionary Computation, vol. 2, no. 3, (1994), pp. 221-248.

[18] K. Deb, A. Pratap, S. Agarwal and T. Meyarivan, "A fast and elitist multiobjective genetic algorithm: NSGA-II, Evolutionary Computation”, IEEE Transactions on, vol. 6, no. 2, April (2002), pp. 182-197. 
[19] E. Zitzler and L. Thiele, "Multiobjective evolutionary algorithms: a comparative case study and the strength Pareto approach, Evolutionary Computation”, IEEE Transactions on, vol. 3, no. 4, (1999) November, pp. 257-271.

[20] E. Zitzler, M. Laumanns and L. Thiele, "SPEA2: improving the strength Pareto evolutionary algorithm", In evolutionary for design, optimization and control with application to an industrial problems (EUROGEN2001), (2002), pp. 95-100.

[21] K. Sindhya, K. Miettinen and K. Deb, "A Hybrid Framework for Evolutionary Multi objective Optimization”, Evolutionary Computation, IEEE Transactions on, vol. PP, no. 99, (2012), pp. 1-1.

[22] J. N. Laneman, D. N. C. Tse and G. W. Wornell, "Cooperative diversity in wireless networks: Efficient protocols and outage behavior", Information Theory, IEEE Transactions on, vol. 50, no.12, (2004) December, pp. 3062-3080.

\section{Authors}

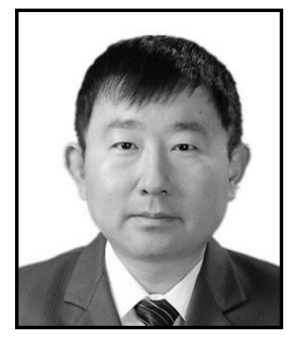

Jizheng Li, (M'07) Work as a Lecture in Harbin University of Science and Technology. Member of Heilongjiang Communication Association. Research direction: Wireless communication and so on.

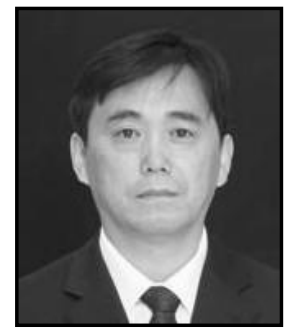

Ming Diao, Work as a Professor in Harbin Engineering University, master\&doctor of engineering tutor.Senior member of China communication association, member of China image and graphics association, Journal of Harbin engineering university staff.

Research direction: broadband signal detection, processing and identification, communication signal processing 
International Journal of Signal Processing, Image Processing and Pattern Recognition Vol. 9, No. 7 (2016) 